\title{
Öber die Adsorption von Elektrolyten und kolloiden Körpern durch Casein.
}

Von

Herman Palme.

Mil zwei Kurvenzeichnungen im Text.

(Aus der biochemischen Abteilung der Hochschule Stockholm.)

(Der Redaktion zugegangen am 10. Juni 1914.)

Auf Anregung von Herrn Professor H. v. Euler sind von mir einige Untersuchungen ausgeführt worden, die den Zweck hatten, die Fähigkeit des Caseins zu studieren, aus Lösungen fester Körper die gelöste Substanz zu adsorbieren. Für die Untersuchungen kamen bisher nur solche Körper in Betracht, in deren Lösungen das Casein praktisch unlöslich war. Von diesen sind Ferrocyanwasserstoffsäure und Kupferacetat eingehender untersucht worden, während die Untersuchungen über das Verhalten des Caseins dem Eisenchlorid und kolloidem Eisenhydroxyd gegenüber fortgesetzt werden.

\section{Darstellung des Caseins.}

$\mathrm{Da}$ es von großer Wichtigkeit war, ein möglichst gleichförmiges und reines Casein zu verwenden, wurde dieses mit großer Sorgfalt bereitet. Besonders mußten Verunreinigungen von Elektrolyten und Fett vermieden werden. Zu diesem Zweck wurde im wesentlichen nach der von Hammarsten angegebenen Methode verfahren. Entrahmte Milch wurde mit der fünffachen Menge Wasser verdünnt und sodann mit sehr verdünnter Essigsäure versetzt, bis ein grobflockiger Niederschlag entstand. Dieser wurde mehrmals durch Dekantieren mit schwach essigsäurehaltigem Wasser gewaschen und dann in sehr verdünntem Ammoniak gelöst, wonach die Lösung geklärt und von neuem mit Essigsäure gefällt wurde. Der Niederschlag wurde alsdann in einem großen Mörser mit Wasser zu einem möglichst gleichförmigen Brei ausgerieben und nach 
dem Ausrühren mit einer größeren Menge W'asser nach dem Absetzen auf einem leinenen Tuche abgepreßt. Dies Verfahren wurde mehrmals wiederholt.

Das letzte Mal wurde mittels einer Schraubenpresse möglichst viel Wasser entfernt, wonach das Casein mit Alkohol zweimal verrieben und abgepreBt wurde. Die so erhaltene Masse wurde so fein wie möglich in einem Mörser zerrieben und nach dem freiwilligen Verdampfen des anhaftenden Alkohols in Extraktionsapparate vom Soxhletschen Typus gebracht. Im Siedegefäße derselben wurde Äther mit gekörntem Chlorcalcium vermischt, um das dem Casein entzogene Wasser und den Alkohol zurückzuhalten. Die Ätherextraktion, die insgesamt wenigstens zwei Tage fortgesetzt wurde, wurde mehrmals unterbrochen, um die nunmehr sehr hart gewordenen Caseinkörner durch Reiben möglichst zu zerkleinern. Wenn das so behandelte Casein zwei Stunden mit trocknem Äther in dem genannten Apparate extrahiert wurde, konnten nach dem Abdestillieren des Äthers nur geringe Spuren von einem fettähnlichem Rückstand nachgewiesen werden. Das Fett vollständig zu extrahieren, war anscheinend unmöglich. Da indessen die Resultate der vorliegenden Untersuchung davon in keinem Maße beeinflußt $z u$ werden scheinen, wurde darauf keine weitere Rücksicht genommen. Beim Verwenden eines mit geringerer Sorgfalt extrahierten Caseins wurden nämlich die gleichen Resultate erhalten. Auch beim Gebrauch eines von Merck bezogenen, mit "Casein nach Hammarsten» bezeichneten Präparats waren die Ergebnisse dieselben. Dagegen war das Nährcasein (Caseinat) des Handels (Riedel) zu diesem Zweck unbrauchbar, was ja zu erwarten war. Um der mühsamen Darstellung größerer Mengen von Casein zu entgehen, wurde bei den späteren Versuchen das Mercksche Casein verwendet, $\mathrm{da}$, wie genannt, kein Unterschied von dem von mir dargestellten $\mathrm{zu}$ bemerken war.

Nach beendeter Extraktion wurde das von mir bereitete Casein bei Zimmertemperatur an der Luft ausgebreitet und dann zunächst einige Stunden im Vakuum über Paraffin gelassen, um noch anhaftenden Äther zu entfernen. Schließlich 
wurde über Schwefelsäure im Vakuum getrocknet. Die Feuchtigkeit wurde in dieser Weise nicht so vollständig entfernt, wie dies beim Trocknen in der Wärme möglich ist, aber das in der eben beschriebenen Weise dargestellte Präparat ist für die fraglichen Versuche weit geeigneter, weil es von der Flüssigkeit viel leichter benetzt wird.

Um das so erhaltene Casein in eine für Adsorptionszwecke geeignete Form zu bringen, war es nötig, dasselbe möglichst fein zu pulverisieren. Deshalb wurde es in kleinen Portionen in einem Mörser gerieben und sodann durch ein Sieb getrieben, das von solcher Feinheit war, daB auf $1 \mathrm{~cm} 40$ Drähte kamen. Durch dieses wurde es zweimal gesiebt.

Das Casein von Merck wurde in der Reinheit, wie es von der Fabrik erhalten wurde, gepulvert und verwendet.

Das von mir dargestellte und wie oben behandelte Casein verlor bei $105^{\circ}$ getrocknet $1,12 \%$ an Gewicht und hinterließ bei Verbrennung 0,07\% Asche. Die entsprechenden Ziffern für das Mercksche waren 1,04, bezw. 0,06\%.

\section{Verhalten des Caseins zu wässerigen Lösungen von Ferrocyan- wasserstoffsäure.}

Daß sich Casein wie ein amphoterer Elektrolyt verhält, und sowohl mit Säuren als Basen Salze bilden kann, ist schon lange bekannt. $\mathrm{Ob}$ die Konstitution dieser Verbindungen denjenigen der einfacheren amphoteren Elektrolyten ähnlich ist, darüber sind verschiedene Ansichten geäußert worden. Ganz interessant ist $z u$ sehen, wie van Slyke und van Slyke ${ }^{1}$ ) es zu beweisen versuchen, daß bei der Behandlung von Casein mit sehr verdünnten Säuren, z. B. Chlorwasserstoffsäure oder Schwefelsäure, keine gewöhnlichen chemischen Verbindungen entstehen, sondern daß die Bildung dieser Verbindungen durch Adsorptionserscheinungen bedingt ist. Neben dieser Möglichkeit haben sie u. a. die Frage diskutiert, ob nicht die Reaktion die eines hydrolytisch gespaltenen Salzes sei, aber indem sie die Voraussetzung machen, daß gar nichts vom Casein oder dessen Säureverbindung in Lösung gehe, haben sie diese Frage

1) Amer. chem. journ., Bd. 38, S. 444, 1907. 
verneinend beantwortet. Robertson ${ }^{1}$ ) hat jedoch gezeigt, daB die Reaktion eben in dieser Weise zu erklären sei, wenn man diese Voraussetzungen nicht einführt, was dadurch gerechtfertigt ist, daß die meisten Säureverbindungen des Ciaseins löslich sind. Alle Kriterien, die von van Slyke und van Slyke als Beweis für die Adsorptionshypothese angeführt worden sind, werden von Roberts on der Annahme eines hydrolysierbaren Salzes angepaßt. Robertson selbst betrachtete anfänglich die Verbindungen von Casein mit sowohl Säuren als Basen wie gewöhnliche Salze eines amphoteren Elektrolyten und hat dasVerhalten «assoziierender» amphoterer Elektrolyte bei Gegenwart von nichtamphoteren Elektrolyten theoretisch behandelt. ${ }^{2}$ ) Bei seinen späteren Untersuchungen hat er die Beobachtung gemacht, daß die Salze des Caseins nur sehr wenig hydrolytisch spaltbar sind, was mit den niedrigen Dissoziationskonstanten nicht im Einklang steht. Deshalb neigt er der Ansicht zu, daß nicht freie $\mathrm{NH}_{2}-$ und $\mathrm{COOH}-G r u p p e n$, sondern die Gruppen - $\mathrm{CO} \cdot \mathrm{NH}-$ bei der Salzbildung teilnehmen. ${ }^{3}$ ) Für die vorliegende Untersuchung interessiert es zunächst nur, annehmen zu können, daß das Salz des Caseins mit Ferrocyanwasserstoffsäure nur in sehr geringem Maße hydrolytisch spaltbar ist, und daß also, wenn es unlöslich angenommen wird, die Zusammensetzung desselben von der Konzentration der umgebenden Flüssigkeit nur wenig beeinflußt wird.

Die von mir ausgeführten Versuche sollen nun beschrieben werden. Für einige vorbereitende Versuche wurde Ferrocyanwasserstoffsäure von mir dargestellt; für die unten zu beschreibenden Versuche wurde aber von Merck bezogenes Präparat verwendet. Die Versuche wurden in der Weise ausgeführt, daß eine gewogene Menge Casein in ein $100 \mathrm{ccm}$ fassendes Schüttelrohr gebracht, mit einer genau gemessenen Menge filtrierter Lösung von bekanntem Gehalt übergossen wurde, wonach in einem Thermostat bei $25^{\circ}$. C. bis zum Eintritt des Gleichgewichts geschüttelt wurde. Nachdem sich

1) Journ. biolog. chem., Bd. 4, S. 35, 1907.

2) Journ. physical chem., Bd. 1, S. 437, 1907.

s) Journ. physical chem., Bd. 15, S. 521, 1911. 
der feste Körper abgesetzt hatte, was sehr leicht geschah, wurde die Lösung durch reinstes Filtrierpapier filtriert, und die Konzentration durch Titrieren mit $n / 10$-Natronlauge ermittelt. Das Gleichgewicht stellte sich ziemlich rasch ein; bei den Versuchen wurde im allgemeinen ungefähr 3 Stunden geschüttelt, was durchaus hinreichend war, wie sich durch besondere Versuche herausstellte. Bei der Zersetzlichkeit der Ferrocyanwasserstoffsäure war zu langwieriges Schütteln zu vermeiden. Bei fast sämtlichen Versuchen waren trotzdem sowohl die Lösung wie die feste Substanz von Berlinerblau mehr oder weniger blaugrün gefärbt, auch ein Geruch nach Cyanwasserstoff war zu bemerken. Allerdings waren die dadurch bedingten Fehler so gering, daß sie beim Titrieren mit zehntel-normaler Lauge nicht merkbar waren. Eine Lösung, die durch langes Stehen recht kräftig blau gefärbt war, erwies bei der Titrierung praktisch denselben Gehalt wie die frisch bereitete.

Zunächst wurde eine Reihe von Versuchen ausgeführt, bei denen $2 \mathrm{~g}$ Casein mit $50 \mathrm{ccm}$ von Lösungen verschiedener Konzentration geschüttelt wurde. Es war ja vorauszusehen, daß der Gleichgewichtszustand nur von der Endkonzentration der umgebenden Lösung abhängig, und die absorbierte Säuremenge bei gleicher Endkonzentration der Lösung der Caseinmenge proportional sei, wenn wir beiläufig die vom Casein der Lösung entzogene Säuremenge als «absorbiert * bezeichnen. Um dies $z u$ bestätigen, wurden einige Versuche ausgeführt, wo sowohl die Caseinmenge wie das Volumen der Lösung variiert wurden. Von den mit $2 \mathrm{~g}$ Casein und $50 \mathrm{ccm}$ Lösung gefundenen Werten wurde eine Kurve gezeichnet, wobei die Endkonzentration der Lösung, in Normalität angegeben, als Abszissen, und die auf $1 \mathrm{~g}$ Casein bezogenen *absorbierten Mengen, in Grammen angegeben, als Ordinaten abgesetzt wurden. Die mit anderen Caseinmengen und Lösungsvolumina erhaltenen Werte stimmen mit der so gezeichneten Kurve sehr gut überein. Da es sich bei der verwendeten Methode um die Bestimmung von Konzentrationsdifferenzen handelt, sind die angegebenen Werte das Mittel von wenigstens drei unter den 
gleichen Bedingungen ausgeführten Versuchen. Die gefundenen Werte sind in der folgenden Tabelle zusammengeführt worden.

Tabelle I.

\begin{tabular}{r|c|c|c|c|c}
\hline \hline Nr. & $\begin{array}{c}\text { Casein- } \\
\text { menge } \\
\text { in } \mathbf{g}\end{array}$ & $\begin{array}{c}\text { Volumen } \\
\text { der } \\
\text { Lösung } \\
\text { in ccm }\end{array}$ & $\begin{array}{c}\text { Konzentration } \\
\text { der Lösung } \\
\text { vor dem } \\
\text { Schülteln }\end{array}$ & $\begin{array}{c}\text { Konzentration } \\
\text { der Lösung } \\
\text { nach dem } \\
\text { Schütteln }\end{array}$ & $\begin{array}{c}\text { Von 1 g Casein } \\
\text { absorbierte } \\
\text { Säuremenge } \\
\text { in mg }\end{array}$ \\
\hline 1 & 2 & 50 & 0,035 & 0,003 & 43,2 \\
2 & 2 & 50 & 0,069 & 0,026 & 58,4 \\
3 & 2 & 50 & 0,172 & 0,117 & 75,2 \\
4 & 2 & 50 & 0,345 & 0,277 & 92,3 \\
5 & 2 & 50 & 0,981 & 0,890 & 123,9 \\
6 & 2 & 50 & 1,963 & 1,848 & 153,9 \\
7 & 2 & 100 & 0,039 & 0,018 & 54,5 \\
8 & 2 & 100 & 0,626 & 0,585 & 112,0 \\
9 & 1 & 100 & 0,228 & 0,212 & $.85,0$ \\
10 & 1 & 75 & 0,499 & 0,464 & 107,2 \\
11 & 2 & 50 & 0,026 & 0,002 & 32,9 \\
12 & 2 & 50 & 0,013 & 0,001 & 17,0
\end{tabular}

Was die Form der Kurve betrifft, ist es auffallend, daß der hintere Teil derselben sich der Ordinatenachse anschmiegt, ohne jedoch dieselbe zu berühren. Daß sich dieser vertikale Teil auf die Bildung eines Salzes zwischen Casein und Ferrocyanwasserstoffsäure bezieht, dürfte außer $Z$ weifel sein. Daß die Kurve in diesem Gebiet mit der Achse nicht völlig zusammenfällt, beruht mit Wahrscheinlichkeit auf geringer Hydrolyse. Wenn man annimmt, daß sowohl freies Casein wie sein ferrocyanwasserstoffsaures Salz unlöslich sind, muß dem Massenwirkungsgesetz gemäß bei der Einwirkung von Säure auf überschüssiges Casein. die Reaktion so lange verlaufen, bis die Konzentration der Säure einen konstanten Wert angenommen hat. Schreibt man nämlich die Reaktionsformel der Hydrolyse in der vereinfachten Form:

Caseinsalz + Wasser $\rightleftarrows$ Freies Casein + Säure, so sieht man sogleich, dạ $B$ unter den genannten Voraussetzungen dies zutreffen muß, denn die Konzentrationen von sowohl dem 
Casein wie seinem Salze, ebenso wie von dem Wasser sind als konstant zu betrachten, wodurch auch die Konzentration der Säure konstant sein muß. Der fragliche Teil der Kurve sollte also der Ordinatenachse parallel sein. $\mathrm{DaB}$ dies nicht ganz genau der Fall ist, kann ja von verschiedenen Umständen abhängen, so z. B. kann man denken, daß die Voraussetzungen über die Unlöslichkeit des Caseins und des Caseinsalzes nicht ganz streng gelten. Die Abweichung ist ja allerdings sehr klein, besonders wern man betrachtet, daß es sich in dem fraglichen Gebiet um Säurekonzentrationen von nur bis zu ungefähr 0,002normal handelt. Als diesem Wert entsprechend kann man wegen des fast vertikalen Verlaufs dieses Kurventeils einen Absorptionswert von 37,8 mg feststellen. Dies entspricht gerade der Menge einer Säure, die nach Long $u$. a. von $1 \mathrm{~g}$ Casein als Salz gebunden wird (7 ccm einer 0,1-normalen Säure).

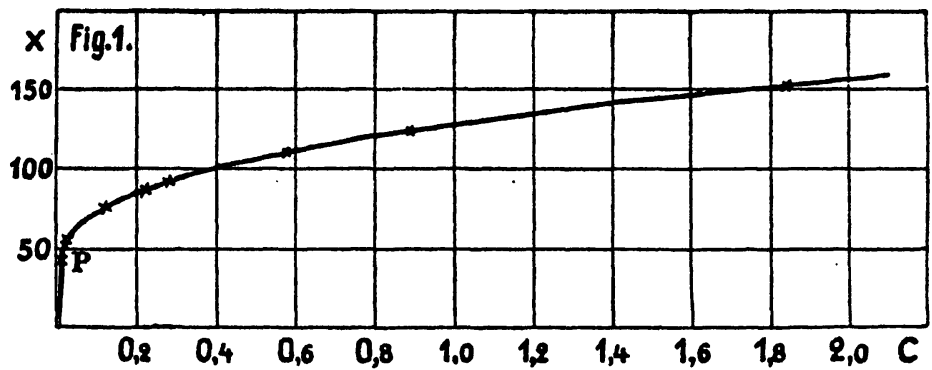

Von etwa da ab weicht die Kurve immer rascher von dem vertikalen Verlauf ab. In diesem Gebiet erweist sie die Ansicht einer gewöhnlichen Adsorptionskurve. Um zu sehen, ob dieser Teil irgend welchen der bisher aufgestellten Adsorptionsformeln anzupassen wäre, wurde im Punkte $\mathrm{P}(\mathrm{C}=0,002, \mathrm{X}=37,8)$ der Ursprung eines neuen, rechtwinkligen Koordinatensystems gelegt, und die Adsorptionsformeln auf die in bezug auf dieses gefundenen Koordinaten geprüft. Wenn wir die bei den Versuchen gefundenen Werte der Säurekonzentrationen mit $\mathrm{c}$ und diejenige der von $1 \mathrm{~g}$ Casein aufgefundenen Säuremengen mit $x$ bezeichnen, so berechnen sich die Koordinaten in dem neuen System $c_{1}=c-0,002$, bezw. $x_{1}=x-37,8$.

Da bei den angewandten Konzentrationen die Kurve keinem ausgeprägten Maximalwert zustrebt, wurde zunächst die von

Hoppe-Seyler's Zeitschrift f. physiol. Chemie. XCII. 
Freundlich ${ }^{1)}$ aufgestellte Formel geprüft, die mit den obigen Bezeichnungen lautet:

$$
\mathrm{x}_{1}=\mathrm{k} \cdot \mathrm{c}_{1}^{\mathrm{n}}
$$

Die Ergebnisse finden sich in der Tabelle II, wo für gegebene Werte von $c$, bezw. $c_{1}$, die Werte von $\mathrm{x}$ und $\mathrm{x}_{1}$ berechnet worden sind. Außerdem ist die Formel von Arrheniu s ${ }^{2}$ ) benutzt worden, wobei ein Näherungswert von s aus den Beobachtungen berechnet worden ist. Die Formel von Arrhenius lautet:

oder integriert

$$
\mathrm{k} \frac{\mathrm{dx}}{\mathrm{dc}}=\frac{\mathrm{s}-\mathrm{x}}{\mathrm{x}}
$$

$$
\log 10 \frac{\mathrm{s}}{\mathrm{s}-\mathrm{x}}-0,4343 \cdot \frac{\mathrm{x}}{\mathrm{s}}=\frac{1}{\mathrm{k}} \cdot \mathrm{c},
$$

wobei in diesem Falle $c$ gegen $c_{1}$ und $x$ gegen $x_{1} z u$ vertauschen sind.

Tabelle II.

\begin{tabular}{c|c|c|r|r|r|r|r}
\hline \hline & & & & \multicolumn{3}{|c}{ Berechnet nach } \\
& $c_{1}$ & x beob. & $\mathrm{x}_{\mathbf{1}}$ beob. & Freundlich & Arrhenius \\
& $\cdot$ & & & $\mathrm{x}$ & $\mathrm{x}_{\mathbf{1}}$ & $\mathrm{x}$ & $\mathrm{x}_{\mathbf{1}}$ \\
\hline 0,0025 & 0,0005 & 43,2 & 5,4 & 41,8 & 4,0 & 43 & 5 \\
0,0184 & 0,0164 & $54, \overline{0}$ & 16,7 & 54,7 & 16,9 & 54 & 16 \\
0,0258 & 0,0238 & 58,4 & 20,6 & 57,4 & 19,6 & 58 & 20 \\
0,1168 & 0,1148 & 75,2 & 37,4 & 75,3 & 37,5 & 77 & 39 \\
0,2766 & 0,2746 & 92,3 & 54,5 & 91,2 & 53,6 & 95 & 57 \\
0,8896 & 0,8876 & 123,9 & 86,1 & 124,5 & 86,7 & 128 & 90 \\
1,8416 & 1,8396 & 153,9 & 116,1 & 154,7, & 116,9 & 152 & 114
\end{tabular}

Die benutzten Konstanten sind bei der Formel von Freundlich $\mathrm{k}=90$ und $\mathrm{n}=0,41$ und bei derjenigen von Arrhenius $\mathrm{k}=6,4$ und $\mathrm{s}=150$. Die berechneten Werte stimmen mit den gefundenen gut überein.

III. Verhalten des Caseins gegen Lösungen von Kupferacetat.

Die Verbindungen des Caseins mit Schwermetallen sind ganz wenig untersucht worden. Durch Fällen von löslichen Caseinsalzen mit Salzen der Schwermetalle werden Verbin-

1) Freundlich, Kapillarchemie, S. 149 (1909).

2). Meddelanden fr. K. Vet. Akad.:s Nobelinstitut, Bd.2, Nr. 7 (1911). 
dungen von sehr variabler Zusammensetzung erhalten. Ebenso ist dies der Fall, wenn festes Casein mit Lösungen solcher Salze behandelt wird. Die Annahme liegt dabei nahe, daß die Entstehung dieserVerbindungen durch Adsorption bedingt ist. Um zu erforschen, ob die fraglichen Verbindungen irgend welchen bekannten Gesetzen der Adsorption anzupassen wären, wurden die folgenden Versuche ausgeführt. In genau derselben Weise wie oben mit Ferrocyanwasserstoffsäure wurde mit Lösungen von neutralem Kupferacetat verfahren. Dabei ergab sich, daß Kupfer aus der Lösung vom Casein adsorbiert wird, und daß für gegebene Konzentration ein bestimmter Gleichgewichtszustand eintritt. Die Ergebnisse wurden graphisch dargestellt, wobei eine stetige Kurve erhalten wurde. Die feste Phase

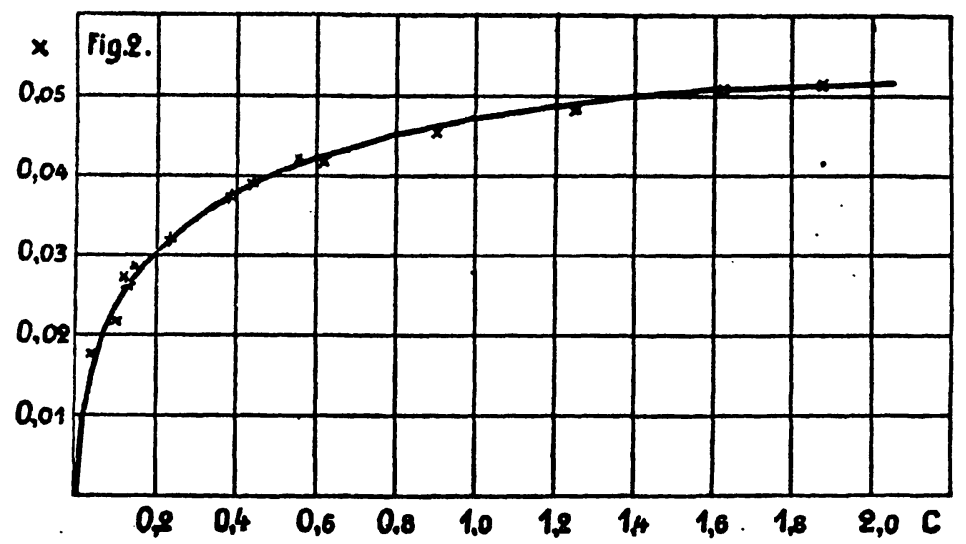

stellte eine hellblaue Verbindung dar. Das Casein war in Kupferacetatlösung praktisch vollkommen unlöslich. Das Gemisch schäumte zwar recht kräftig, was auf einen kleinen Caseingehalt deutet, aber an eine quantitative Bestimmung der gelösten Caseinmenge war nicht zu denken.

Das angewandte Kupferacetat wurde zunächst auf richtige Zusammensetzung geprüft. Das Kupfer wurde in diesem wie in allen übrigen Fällen elektrolytisch bestimmt und zwar mittels Quecksilberkathode unter Benutzung des Fraryschen Apparats ${ }^{1}$ ) für elektromagnetische Rührung. Es bietet diese Methode die Vorteile einer sowohl genauen wie schnellen Ausführung. Die

1) Zeitschrift f. Elektrochemie, Bd. 23, S. 308 (1907). 
Essigsäure wurde teils durch Destillation nach Zusatz von Phosphorsäure bestimmt und teils dadurch, daß das Kupfer mit Schwefelwasserstoff gefällt und ein aliquoter Teil der von Kupfersulfid durch Filtrieren befreiten Flüssigkeit mit $\mathrm{n} /$ 10- $^{-}$ Natronlauge titriert wurde. Beide Methoden ergaben genau übereinstimmende Werte, und zwar entsprach $1,0 \mathrm{~g}$ Kupfer im Mittel 1,866 g Essigsäure, während der theoretisch berechnete Wert $1,888 \mathrm{~g}$ beträgt. Das so befundene Präparat wurde als richtig angesehen und für die folgenden Versuche verwendet.

Eine Frage, die von großem Interesse ist, nämlich diejenige, wie die Reaktion qualitativ verläuft, wurde später untersucht, soll aber hier beschrieben werden. Da es wahrscheinlich war, daß die entstandene Verbindung durch Adsorption entstehe, fragte es sich, ob das ganze Kupferacetatmolekül oder nur der basische Bestandteil desselben adsorbiert wurden, oder auch ob der basische und der saure Bestandteil in verschiedenem Maße aufgenommen wurden. Zur Entscheidung dieser Frage wurden $2 \mathrm{~g}$ Casein mit $100 \mathrm{ccm}$ Kupferacetatlösung geschüttelt und nach dem Entstehen des Gleichgewichts sowohl die Kupfer- wie die Essigsäurekonzentration bestimmt. Die folgende Tabelle gibt die Resultate an.

Tabelle III.

\begin{tabular}{c|c|c|c|c|c}
\hline \multirow{2}{*}{$\begin{array}{c}\text { Cu-Menge } \\
\text { zugesetzt }\end{array}$} & $\begin{array}{c}\text { Cu-Menge } \\
\text { gefunden } \\
\text { nach der } \\
\text { Adsorption }\end{array}$ & \multicolumn{2}{|c|}{ Essigsäuremenge } & \multicolumn{2}{|c}{ Adsorbiert in \% } \\
\hline 0,5200 & 0,4420 & 0,9807 & 0,9778 & 15,0 & 3,0 \\
0,2076 & 0,1509 & 0,3826 & 0,3542 & 27,3 & 7,3 \\
\end{tabular}

Wie aus diesen Versuchen hervorgeht, wird aus der Lösung viel mehr Kupfer als Essigsäure entfernt. Es liegt ja dabei nahe, anzunehmen, daß es Kupferoxyd ist; das adsorbiert wird, welches der Hydrolyse zufolge sich in der Lösung kolloidal befindet.

Unter solchen Umständen war es nicht wie bei der Ferrocyanwasserstoffsäure a priori klar, daß der Gleichgewichts- 
zustand von der absoluten Menge der Bestandteile des Syștems unabhängig sei, was aus der folgenden Überlegung hervorgeht. Wir nehmen beiläufig an, daß dieselben Gesetze, die für die Adsorption der Ferrocyanwasserstoffsäure galten, auch für diejenige des Kupferhydroxyds zutreffen. Wenn z. B. zu 2 g Casein das eine Mal $50 \mathrm{ccm}$ Lösung zugefügt wird, die auf $100 \mathrm{ccm}$ $0,66 \mathrm{~g} \mathrm{Cu}$ und 1,25 $\mathrm{g}$ Essigsäure enthält, so wird aus dieser etwa $0,08 \mathrm{~g} \mathrm{Cu}$ adsorbiert, so daß die Kupferkonzentration der Lösung nach dem Eintreten des Gleichgewichts $0,50 \mathrm{~g}$ auf $100 \mathrm{ccm}$ beträgt. Die Essigsäurekonzentration wird nur wenig geändert. Ein anderes Mal fügen wir $100 \mathrm{ccm}$ einer Lösung $\mathrm{zu}$, die $0,58 \mathrm{~g} \mathrm{Cu}$ enthält und somit rund 1,10 $\mathrm{g}$ Essigsäure, so wird beim Gleichgewicht $0,08 \mathrm{~g} \mathrm{Cu}$ adsorbiert sein, und die $\mathrm{Cu}$-Konzentration der umgebenden Lösung auf $100 \mathrm{ccm}$ $0,50 \mathrm{~g}$ betragen. Auch in diesem Falle wird die Essigsäurekonzentration nur wenig geändert, oder wenigstens um denselben Betrag wie im vorigen Falle. Also wird beim Gleichgewicht, wenn die Voraussetzungen gelten sollen, im ersteren Falle die Essigsäurekonzentration höher als im letzteren sein. Das System besitzt ja einen Bestandteil mehr als wie bei der Ferrocyanwasserstoffsäure, und das Gleichgewicht muß also von der Konzentration desselben abhängig sein. Indessen geht aus den ausgeführten Versuchen hervor, daß in den Gebieten, die für diese Untersuchungen in Frage gekommen sind, die verschiedene Konzentration der Essigsäure keine merkbare Rolle spielt. Allerdings liegen die davon verursachten Abweichungen innerhalb der Versuchsfehler, wie aus der unten folgenden Tabelle ersichtlich ist.

Zunächst wurde eine Reihe von Versuchen ausgeführt, bei denen $2 \mathrm{~g}$ Casein mit $50 \mathrm{ccm}$ von Kupferacetatlösungen verschiedener Konzentrationen geschüttelt wurden. Dies wurde bei $25^{\circ}$ in einem mit Rührwerk versehenen Thermostaten bewerkstelligt. Das Gleichgewicht stellte sich rasch ein. Zwar wurden keine genauen Zeitmessungen ausgeführt, aber nach 3 Stunden war das Gleichgewicht erreicht. Wenn die Digestion bis auf 24 Stunden ausgedehnt wurde, wirkte dies in keiner Weise auf das Gleichgewicht ein. Sodann wurden Versuche 
mit $2 \mathrm{~g}$ C'asein und $100 \mathrm{ccm}$ Lösung und zuletzt mit $1 \mathrm{~g}$ Casein und $100 \mathrm{ccm}$ Lösung ausgeführt, um zu konstatieren, ob das Gleichgewicht nur von der Kupferkonzentration abhängig sei, und daß bei gleicher Endkonzentration die adsorbierte Kupfermenge der Caseinmenge proportional sei. In der folgenden Tabelle sind die Ergebnisse zusammengefaßt worden. Der Einfachheit und Übersichtlichkeit halber sind die Konzentrationen in Grammen Kupfer pro $100 \mathrm{ccm}$ und die adsorbierten Mengen in Grammen Kupfer pro $1 \mathrm{~g}$ Casein angegeben.

Tabelle IV.

\begin{tabular}{c|c|c|c|c}
\hline \hline $\begin{array}{c}\text { Gesamte } \\
\text { zugesetzte } \\
\text { Cu-Menge }\end{array}$ & Adsorbierte & $\begin{array}{c}\text { Gelöstes Cu } \\
\text { nach } \\
\text { dem Gleich-Menge } \\
\text { gewicht }\end{array}$ & $\begin{array}{c}\text { Cu-Konzentration } \\
\text { in g pro 100 ccm } \\
\text { nach } \\
\text { Gleichgewicht }\end{array}$ & $\begin{array}{c}\text { Von 1 g } \\
\text { Casein } \\
\text { adsorbiertes } \\
\text { Cu }\end{array}$ \\
\hline
\end{tabular}

I. $2 \mathrm{~g}$ Casein und $50 \mathrm{ccm}$ Lösung.

\begin{tabular}{l|l|l|l|l}
0,0545 & 0,0345 & 0,0200 & 0,0400 & 0,0173 \\
0,0909 & 0,0436 & 0,0473 & 0,0946 & 0,0218 \\
0,1363 & 0,0540 & 0,0823 & 0,1646 & 0,0270 \\
0,1817 & 0,0640 & 0,1177 & 0,2354 & 0,0320 \\
0,2726 & 0,0751 & 0,1975 & 0,3950 & 0,0376 \\
0,3634 & 0,0834 & 0,2800 & 0,5600 & 0,0417 \\
0,5450 & 0,0915 & 0,4535 & 0,9070 & 0,0458 \\
0,7267 & 0,0974 & 0,6293 & 1,2586 & 0,0487 \\
0,9084 & 0,1014 & 0,8070 & 1,6140 & 0,0507 \\
0,0400 & 0,1020 & 0,9380 & 1,8760 & 0,0510
\end{tabular}

II. $2 \mathrm{~g}$ Caseịn und $100 \mathrm{ccm}$ Lö̀sung.

\begin{tabular}{l|l|l|l|l}
0,5200 & 0,0780 & 0,4420 & 0,4420 & 0,0390 \\
0,2076 & 0,0567 & 0,1509 & 0,1509 & 0,0284
\end{tabular}

III. $1 \mathrm{~g}$ Gasein und $100 \mathrm{ccm}$ Lösung.

$$
\begin{array}{l|l|l|l|l}
0,6518 & 0,0415 & 0,6103 & 0,6103 & 0,0415 \\
0,1560 & 0,0260 & 0,1300 & 0,1300 & 0,0260
\end{array}
$$

Wenn die in den beiden letzten Kolonnen befindlichen Werte auf ein rechtwinkliges Koordinatensystem bezogen werden, läßt sich, bei der großen Anzahl derselben, die Kurve ganz leicht zeichnen, wobei die Beobachtungsfehler nach Schätzung 
auszugleichen sind. Dabei entsteht eine stetige Kurve ohne Wendepunkte, die sich asymptotisch einer der Abszissenachse parallelen Geraden nähert. Der so gezeichneten Kurve wurden Koordinatenpaare entnommen, und mit diesen Werten wurde sodann geprüft, ob der Kurve die Adsorptionsformel von Arrhenius ${ }^{1}$ ) oder die von Schmidt ${ }^{2}$ ) anzupassen wäre. $\mathrm{Da}$ in diesen beiden Formeln eine Konstante $S$ vorkommt, welche die maximal adsorbierte Menge angibt, mußte, da die Kurve ein Maximum nicht ganz erreicht, ein approximativer Wert desselben berechnet werden. Bei Verwendung der Arrheniusschen Formel wurde dieser Wert $z \mathbf{u} \mathbf{x}=0,0526$ ermittelt. Setzt man diesen Wert in die Formel ein, so berechnet sich für die fraglichen Wertpaare von $c$ und $x$ die Konstante $k$, und gestalten sich die Resultate, wie in der nachfolgenden Tabelle ersichtlich ist. In der Schmidtschen Formel, die in der Form

gegeben ist, wo

$$
\frac{a-x}{v} S=K \times e^{\frac{A(S-x)}{S}}
$$

$x$ die adsorbierte Menge,

a die zugesetzte Menge gelöster Substanz,

$\mathbf{v}$ das Volumen (also $\frac{a-x}{v}$ die Konzentration),

$S$ das Adsorptionsmaximum

bedeuten, gibt es außerdem noch zwei Konstanten, A und K, die willkührlich gewählt werden müssen. Es stellte sich heraus, daß zwar der ganze Verlauf der Kurve dieser Formel nicht gut angepaßt werden kann, aber zum Teil stimmen die gefundenen Werte mit den berechneten recht gut überein, wenn man $A=-3,8$ setzt und sodann $K$ berechnet. Die gefundenen Werte sind in der letzten Kolonne der umstehenden Tabelle zu finden.

Da die Konstanten für ganz geringe Abweichungen recht stark schwanken, sind in der folgenden Tabelle die $\mathrm{zu}$ den gegebenen Konzentrationswerten gehörigen Adsorptionswerte

1) loc. cit.

2) Zeitschrift f. physikal. Chem., Bd. 77, S. 641 (1911). 
Tabelle V.

\begin{tabular}{c|c|c|c}
\hline $\begin{array}{c}\text { Konzentration } \\
\text { der Lösung } \\
\text { in g pro 100 ccm }\end{array}$ & $\begin{array}{c}\text { Adsorbierte } \\
\text { Menge in } \\
\text { pro } \\
\mathbf{1} \text { Casein }\end{array}$ & $\begin{array}{c}\mathrm{K} \text { nach der } \\
\text { Formel } \\
\text { von Arrhenius } \\
\mathbf{S}=0,0526\end{array}$ & $\begin{array}{c}\text { K nach der Formel } \\
\text { von Sch midt } \\
\mathrm{S}=0,0526 \\
\mathrm{~A}=-3,8\end{array}$ \\
\hline 0,05 & 0,0170 & 1,71 & 2,01 \\
0,10 & 0,0233 & 1,62 & 1,87 \\
0,15 & 0,0275 & 1,59 & 1,76 \\
0,20 & 0,0304 & 1,62 & 1,72 \\
0,30 & 0,0346 & 1,67 & 1,67 \\
0,40 & 0,0378 & 1,57 & 1,62 \\
0,50 & 0,0401 & 1,71 & 1,62 \\
0,75 & 0,0442 & 1,74 & 1,64 \\
1,00 & 0,0469 & 1,73 & 1,69 \\
1,25 & 0,0488 & 1,69 & 1,77 \\
1,50 & 0,0501 & 1,65 & 1,89 \\
1,75 & 0,0510 & 1,60 & 2,03 \\
2,00 & 0,0515 & 1,59 & 2,21 \\
\end{tabular}

berechnet unter Benutzung solcher Werte der Konstanten, daß die Abweichungen möglichst klein werden. Zunächst wurden c-Werte als Funktionen von $x$ berechnet, und sodann aus der nach diesen Werten gezeichneten Kurve diejenige der inversen Funktion genommen. Dabei wurden folgende Werte erhalten. Die angewandten Konstanten sind in der nebenstehenden Tabelle angegeben.

Wie aus dieser Tabelle ersichtlich ist, stimmen die gefundenen Werte mit denen nach der.Formel von Arrhenius berechneten in glänzendster Weise überein. Auch die Schmidtsche Formel paßt ganz gut, nur bei denjenigen Werten, die sich dem Adsorptionsmaximum nähern, ist sie nicht brauchbar. Daß so der Fall sein muß, geht aus der folgenden Überlegung deutlich hervor. Der Wert von $\mathrm{K}$ muß notwendig, wenn die Kurve dạs Maximum erreicht, dem Wert der diesem zugehörenden Konzentration gleich sein. Denn wenn $\mathrm{x}=\mathrm{S}$ gesetzt wird, reduziert sich die Adsorptionsformel $z \mathrm{u} K=\mathrm{c}$, wenn $\mathrm{c}=\frac{\mathrm{a}-\mathrm{x}}{\mathrm{v}}$ ist. $\mathrm{Da}$ in dieser Untersuchung das Adsorptionsmaximum noch 
Adsorption von Elektrolyten und kolloiden Körpern durch Casein. 191

Tabelle VI.

\begin{tabular}{|c|c|c|c|}
\hline \multirow{2}{*}{$\begin{array}{l}\text { Konzentration } \\
\text { der Lösung (G) } \\
\text { in } \mathrm{g} \mathrm{Cu} \\
\text { pro } 100 \mathrm{ccm}\end{array}$} & \multirow{2}{*}{$\begin{array}{c}\text { Adsorbierte } \\
\text { Menge Cu in } \mathbf{g} \\
\text { pro } 1 \mathrm{~g} \\
\text { Casein (X) } \\
\text { Gefunden }\end{array}$} & \multicolumn{2}{|c|}{$\begin{array}{c}\text { Adsorbierte Menge } \mathrm{Cu} \text { in } \mathrm{g} \text { pro } 1 \mathrm{~g} \\
\text { Casein berechnet nach der Formel } \\
\text { von }\end{array}$} \\
\hline & & $\begin{array}{l}\text { Arrhenius } \\
\mathrm{S}=0,0526 \\
\mathrm{~K}=1,65\end{array}$ & $\begin{array}{l}\text { Schmidt } \\
\mathrm{S}=0,0526 \\
\mathrm{~K}=1,81 \\
\mathrm{~A}=-3,8\end{array}$ \\
\hline 0,05 & 0,0170 & 0,0173 & 0,0183 \\
\hline 0,10 & 0,0233 & 0,0231 & 0,0240 \\
\hline 0,15 & 0,0275 & 0,0270 & 0,0278 \\
\hline 0,20 & 0,0304 & 0,0301 & 0,0304 \\
\hline 0,30 & 0,0346 & 0,0347 & $0,03 \notin 1$ \\
\hline 0,40 & 0,0378 & 0,0380 & 0,0368 \\
\hline 0,50 & 0,0401 & 0,0405 & 0,0392 \\
\hline 0,75 & 0,0442 & 0,0448 & 0,0435 \\
\hline 1,00 & 0,0469 & 0,0473 & 0,0469 \\
\hline 1,25 & 0,0488 & 0,0490 & 0,0488 \\
\hline 1,50 & 0,0501 & 0,0501 & 0,0505 \\
\hline 1,75 & 0,0510 & $\cdot 0,0510$ & - \\
\hline 2,00 & 0,0515 & 0,0514 & - \\
\hline
\end{tabular}

bei $c=2,0$ nicht erreicht ist, sollte $K$ noch größer sein. Wenn dies der Fall wäre, wobei natürlich auch $\mathrm{A}$ einen anderen Wert bekäme, würde die Übereinstimmung viel mangelhafter sein.

Die Formel von Freundlich ist hier nicht geprüft worden, weil diese den asymptotischen Verlauf der Kurve auszudrücken nicht imstande ist.

IV. Verhalten Des Caseins zu wässerigen Lösungen von Eisenchlorid und kolloidem Eisenhydroxyd.

Zunächst wurden Versuche ausgeführt, um die Adsorption von kolloidem Eisenhydroxyd durch Casein zu studieren. Da indessen die Adsorption sehr gering gefunden wurde und kolloides Eisenhydroxyd nicht rein käuflich zu beziehen war, wurden die Versuche vorläufig unterbrochen. Statt dessen wurde Eisenoxychloridlösung (D. A. B. 5) verwendet, wobei die Adsorption einen beträchtlich größeren Wert annahm. Sodann 
wurde mit Lösungen von Eisenchlorid und endlich mit Gemischen von Eisenchlorid- und Eisenoxychloridlösungen gearbeitet. Sämtliche Lösungen, außer denjenigen von kolloidem Eisenbydroxyd, enthalten ja neben kolloid gelösten Stoffen noch Elektrolyte. Bei den bisher ausgeführten Versuchen hat sich ergeben, daß, wenn die Lösung von Elektrolyten frei ist, die Adsorption äußerst gering ausfällt, wogegen in dem Falle, daß Elektrolyte zugegen sind, die Adsorption recht beträchtliche Werte annimmt. Den größten Betrag erreichte sie bei den Gemischen von Eisenchlorid und Eisenoxychlorid. Diese Ergebnisse sind in voller Analogie mit den Beobachtungen von Bayliss, ${ }^{1}$ ) der bei seinen Untersuchungen über die Adsorption von Farbstoffen in Filtrierpapier die Adsorption um so geringer fand, je reiner das Papier war, und die Vermutung aussprach, daß, wenn es gelänge, alle Elektrolyte vollständig zu entfernen, die Adsorption gleich Null sein würde. Sogar durch ganz geringe Mengen von Elektrolyten wurde das Adsorptionsvermögen beträchtlich erhöht. Auch bei den eben besprochenen, von mir ausgeführten Versuchen ist zu bemerken, daß viel mehr Casein in die Lösung ging, als dies bei den Versuchen mit Ferrocyanwasserstoffsäure und Kupferacetat der Fall war, wodurch das Gleichgewicht viel komplizierter wird. Die Untersuchungen hierüber werden fortgesetzt. ${ }^{2}$ )

1) Bio-chem. journ., Bd. 1, S. 175 (1906).

2) Außer den oben beschriebenen Versuchen sind einige in derselben Weise mit Alkalisalzen von verschiedenen Säuren ausgeführt worden. Dabei hat nur konstatiert werden können, daß sich Casein wie ein gewöhnlicher Elektrolyt veṛhält, indem es schwächere Säuren, wie z. B. Essigsäure und Salicylsäure, aus seinen Salzen freimacht, was ja schon lange bekannt war. $\mathrm{Ob}$ auch die basische Komponente des Salzes mit dem Casein in Reaktion tritt, hat. mit Sicherheit nicht nachgewiesen werden können, aber dies ist ja wegen des amphoteren Charakters des Caseins nicht unwahrscheinlich. Alle Alkalisalze von schwachen Säuren scheinen Casein in höherem oder geringerem Maße zu lösen. Durch die leichte Löslichkeit des Caseins in solchen Salzlösungen wird das Studium der Gleichgewichtsverhältnisse sehr erschwert. Sogar durch Kaliumferrocyanid wird es ziemlich leicht gelöst, trotzdem es in der freien Säure ganz unlöslich ist. 
Adsorption von Elektrolyten und kolloiden Körpern durch Casein. 193

\section{Zusammenfassung.}

1. Zunächst ist die von mir angewandte Darstellungsmethode des Caseins beschrieben worden, die im wesentlichen derjenigen von Hammarsten ähnlich ist.

2. Es ist die Einwirkung von gelöster Ferrocyanwasserstoffsäure auf festes Casein näher untersucht worden. Bei der Bindung der gelösten Säure durch das Casein machen sich zweifellos die chemischen Valenzen des Caseins geltend. Die Kurve stellt nämlich bei niedrigen Säurekonzentrationen die eines hydrolysierbaren Salzes dar und nimmt von einem gewissen Punkte ab die Form einer Adsorptionskurve an, die sich sowohl der Adsorptionsformel von Arrhenius als derjenigen von Freundlich gut anpassen läßt.

3. Alsdann ist die Adsorption von Kupfer aus gelöstem Kupferacetat durch festes Casein studiert worden. Die Adsorptionskurve schließt sich der Formel von Arrhenius ausgezeichnet an, und auch die von Schmidt ist für den größten Teil ihres Verlaufs gut verwendbar.

4. Einige vorbereitende Versuche über die Adsorption durch Casein aus Lösungen von kolloidem Eisenhydroxyd sowie von Eisenoxychlorid und Eisenchlorid sind ausgeführt worden, und es hat sich dabei herausgestellt, daß die Adsorption um so geringer ist, je weniger Elektrolyte die Lösung enthält.

Stockholm, im Mai 1914. 